\title{
ON DIFFERENCE METHODS FOR THE SOLUTION OF A TRICOMI PROBLEM( $\left.{ }^{1}\right)$

\author{
BY \\ HAJIMU OGAWA
}

1. Introduction. The purpose of this paper is the investigation of approximate solutions to a Tricomi problem for the partial differential equation

$$
L u=K(y) u_{x x}+u_{y y}+a(x, y) u_{x}+b(x, y) u_{y}+c(x, y) u=f(x, y),
$$

where $K$ is a continuous function which is positive for $y$ positive and negative for $y$ negative. Thus the equation is elliptic, parabolic or hyperbolic according as $y>0, y=0$ or $y<0$.

For $y<0$ the equation (1.1) has real characteristics given by the two families

$$
\begin{aligned}
& d y / d x=(-K)^{-1 / 2}, \\
& d y / d x=-(-K)^{-1 / 2} .
\end{aligned}
$$

Let $A$ and $B$ be two points on the $x$-axis with $x_{A}<x_{B}$. By $D$ we denote the open domain bounded by the characteristic $\Gamma_{1}$ of the family (1.2b) passing through the point $A$, the characteristic $\Gamma_{2}$ of the family (1.2a) passing through the point $B$, and by the simple arc $\Gamma^{+}$in the upper half-plane with endpoints at $A$ and $B$. (We will consider only bounded domains.)

The Tricomi problem for the equation (1.1) on $D$ is the problem of finding a function $u$, continuous in $\bar{D}$, which satisfies the equation (1.1) in $D$, and which takes on the boundary values

$$
u=\phi_{1} \text { on } \Gamma^{+}, \quad u=\phi_{2} \text { on } \Gamma_{1},
$$

where $\phi_{1}$ and $\phi_{2}$ are given functions. Such a problem was first solved by Tricomi [1] for the case $K(y)=y, a \equiv b \equiv c \equiv f \equiv 0$.

We approximate (1.1) by a difference equation

$$
L_{h} U=f
$$

in a function $U$ which is defined on a mesh region $D_{h}$ depending on the original domain $D$. The problem of solving the differential equation (1.1) with the boundary conditions (1.3) is replaced by the problem of solving the difference equation (1.4) with suitable boundary conditions, and investigating the behavior of $U$ as the mesh size tends to zero. Filippov [2] proved that if the Tricomi problem for the equation

Presented to the Society, April 22, 1961; received by the editors December 29, 1960.

(1) This paper is part of a doctoral dissertation presented at the University of California, Berkeley. The author is indebted to his thesis director, Professor M. H. Protter, for his valuable advice and encouragement. 


$$
y u_{x x}+u_{y y}=f(x, y)
$$

has a solution $u$ in $D$ which is sufficiently smooth, then the solution to an appropriate difference equation converges to $u$ as the mesh size tends to zero. The techniques used in this paper, as in [2], depend essentially on determining under what conditions the solution of the difference equation satisfies a maximum principle, in analogy with the maximum principle for the differential equation. The latter may be stated in the following form: if $L u \geqq 0$ in $D$ and $u$ is a nondecreasing function of $y$ on $\Gamma_{1}$, then the maximum of $u$ on $\bar{D}$, if non-negative, is attained on $\Gamma^{+}$. Such a principle was first discovered by Germain and Bader [3] for the Tricomi equation

$$
y u_{x x}+u_{y y}=0
$$

and was extended by Agmon, Nirenberg and Protter [4] to the equation (1.1) with the coefficients satisfying a complicated set of inequalities. In the present paper, we find that solutions to the difference equation have the maximum property provided that the coefficients of the difference equation satisfy certain inequalities which are consequences of the conditions of [4] away from the $x$-axis. However, near the $x$-axis, conditions in addition to those of [4] are necessary in order that the difference equation have the maximum property.

Using the maximum principle we prove that, for a sufficiently fine mesh, the difference equation has a unique solution $U$ for arbitrary functions $f, \phi_{1}$ and $\phi_{2}$, and that $U$ converges to the solution $u$ of the differential equation (1.1) with boundary conditions (1.3), provided that $u$ exists and is twice continuously differentiable in $\bar{D}$. In $\$ 6$ we prove that under certain restrictions on $K$ near the $x$-axis, and on the domain $D^{+}$, the part of $D$ for which $y \geqq 0$, the regularity conditions on the derivatives of $u$ at the boundary of the region may be weakened.

2. The difference problem. We assume that $K$ is of class $C^{3}(\bar{D})$ with $K(y)>0$ for $y>0$ and $K(y)<0$ and $K^{\prime}(y)>0$ for $y<0$. Furthermore, we suppose that $a$ and $b$ are of class $C^{1}(\bar{D})$ and $c$ and $f$ are of class $C(\bar{D})$.

Integrating the equations (1.2) with respect to $y$, we obtain the characteristics of the differential equation in the form $x-x_{0}= \pm G(y)$, where

$$
G(y)=\int_{y}^{0}[-K(\eta)]^{1 / 2} d \eta
$$

We divide the segment $A B$ into $N$ equal parts, each of length $h$, and through each of the points $x_{k}=x_{A}+k h(k=1,2, \cdots, N-1)$ we draw the characteristics

$$
x-x_{k}= \pm G(y) \text {. }
$$

These characteristics, together with the characteristics $\Gamma_{1}$ and $\Gamma_{2}$, intersect at the points 


$$
\left(x_{A}+\frac{n h}{2}+k h,-y_{n}\right) ; k=0,1, \cdots, N-n ; n=1,2, \cdots, N
$$

with the ordinates satisfying

$$
G\left(-y_{n}\right)=n h / 2 ; \quad n=1,2, \cdots, N .
$$

Taking for $y<0$ the points given by (2.2), and for $y \geqq 0$ the points of the form $\left(x_{A}+k h, m h\right)(k, m$ integers) which lie in $\bar{D}$, we obtain a mesh region $\bar{D}_{h}$. For $y>0$, we define the neighbors of the mesh point $(x, y)$ to be the four mesh points $(x+h, y),(x-h, y),(x, y+h)$ and $(x, y-h)$. We call the boundary $\Gamma_{h}$ of $\bar{D}_{h}$ those points of $\bar{D}_{h}$ in the upper half-plane for which not all four neighboring points belong to $\bar{D}_{h}$, together with all points of $\bar{D}_{h}$ which lie on $\Gamma_{1}$, and the point $B$. The totality of points of $\bar{D}_{h}$ which are not boundary points we call the interior mesh region $D_{h}$. Let $D_{h}^{-}$and $D_{h}^{+}$consist of all points of $D_{h}$ for which $y<0$ and $y>0$, respectively, and let $\Gamma_{h}^{-}$and $\Gamma_{h}^{+}$be the points of $\Gamma_{h}$ for which $y<0$ and $y \geqq 0$, respectively. Finally we let $\gamma_{h}$ be the points in $D_{h}$ for which $y=0$.

We now introduce a difference operator $L_{h}$ which acts on any function $U$ defined on $\bar{D}_{h}$. For any point $\left(x,-y_{n}\right)$ of $D_{h}^{-}$we define

$$
\begin{aligned}
& L_{h} U\left(x,-y_{n}\right)=\frac{1}{\lambda_{n} \lambda_{n+1}}\left\{\frac{2 \lambda_{n+1}}{\lambda_{n}+\lambda_{n+1}} U\left(x-\frac{h}{2},-y_{n-1}\right)\right. \\
& \left.\quad+\frac{2 \lambda_{n}}{\lambda_{n}+\lambda_{n+1}} U\left(x-\frac{h}{2},-y_{n+1}\right)-U\left(x-h,-y_{n}\right)-U\left(x,-y_{n}\right)\right\} \\
& \quad+a\left(x,-y_{n}\right) \frac{1}{h}\left\{U\left(x,-y_{n}\right)-U\left(x-h,-y_{n}\right)\right\} \\
& \quad+b\left(x,-y_{n}\right) \frac{1}{\lambda_{n}+\lambda_{n+1}}\left\{U\left(x-\frac{h}{2},-y_{n-1}\right)-U\left(x-\frac{h}{2},-y_{n+1}\right)\right\} \\
& \quad+c\left(x,-y_{n}\right) U\left(x,-y_{n}\right),
\end{aligned}
$$

where $\lambda_{n}=y_{n}-y_{n-1}$ and $y_{0}=0$. At a point $(x, y)$ of $D_{n}^{+}$we let

$$
\begin{aligned}
L_{h} U(x, y)= & K(y) \frac{1}{h^{2}}\{U(x-h, y)-2 U(x, y)+U(x+h, y)\} \\
& +\frac{1}{h^{2}}\{U(x, y-h)-2 U(x, y)+U(x, y+h)\} \\
& +a(x, y) \frac{1}{h}\{U(x, y)-U(x-h, y)\} \\
& +b(x, y) \frac{1}{2 h}\{U(x, y+h)-U(x, y-h)\}+c(x, y) U(x, y) .
\end{aligned}
$$


and at a point $(x, 0)$ of $\gamma_{h}$ we define

$$
\begin{aligned}
L_{h} U(x, 0)= & \frac{2}{h y_{2}}\left\{\frac{y_{2}}{h+y_{2}} U(x, h)+\frac{h}{h+y_{2}} U\left(x,-y_{2}\right)-U(x, 0)\right\} \\
& +a(x, 0) \frac{1}{h}\{U(x, 0)-U(x-h, 0)\} \\
& +b(x, 0) \frac{1}{h+y_{2}}\left\{U(x, h)-U\left(x,-y_{2}\right)\right\}+c(x, 0) U(x, 0) .
\end{aligned}
$$

The problem of finding the solution $u$ of the differential equation (1.1) in $D$ subject to the boundary conditions (1.3) is replaced by the problem of finding the solution $U$ of the difference equation

$$
L_{h} U=f
$$

on the region $D_{h}$, which satisfies the boundary conditions

$$
\begin{aligned}
& U=\phi_{1} \text { on } \Gamma_{h}^{+}, \\
& U=\phi_{2} \text { on } \Gamma_{h}^{-}
\end{aligned}
$$

We have assumed here that $\phi_{1}$ is a function which is defined and continuous on the domain $\bar{D}^{+}=\{(x, y) \in \bar{D} \mid y \geqq 0\}$. The equations (2.6) and (2.7) form a system of linear algebraic equations in the unknown values of $U$ at the points of $D_{h}$, in which the number of equations is equal to the number of unknowns.

The following result establishes a relation between the difference operator $L$, and the differential operator $L$ for functions of class $C^{2}(\bar{D})$. $h \rightarrow 0$.

THEOREM 2.1. Let $u$ be of class $C^{2}(\bar{D})$. Then on $D_{h}, L_{h} u \rightarrow L u$ uniformly as

Proof. Using Taylor's theorem in a neighborhood of the point $\left(x,-y_{n}\right)$ of $D_{h}^{-}$, we find from (1.1) and (2.3) that for $y<0$,

$$
\begin{aligned}
\left|L u-L_{h} u\right| \leqq & K\left(-y_{n}\right)+\frac{h^{2}}{4 \lambda_{n} \lambda_{n+1}}|| u_{x x} \mid+\frac{h^{2}}{2 \lambda_{n} \lambda_{n+1}} \epsilon_{1}+\frac{2 h}{\lambda_{n}+\lambda_{n+1}} \epsilon_{2}+\epsilon_{3} \\
& +\frac{h}{2}\left\{|a|+\frac{h}{2\left(\lambda_{n}+\lambda_{n+1}\right)}|b|\right\}\left|\bar{u}_{x x}\right|+\frac{h}{2}|b|\left|\bar{u}_{x y}\right| \\
& +\frac{\lambda_{n}^{2}+\lambda_{n+1}^{2}}{2\left(\lambda_{n}+\lambda_{n+1}\right)}|b|\left|\bar{u}_{y y}\right|
\end{aligned}
$$

where all unbarred functions are evaluated at $\left(x,-y_{n}\right)$, and the barred partial derivatives denote values of the functions at points $(\bar{x}, \bar{y})$ which satisfy $x-h<\bar{x}<x,-y_{n+1}<\bar{y}<-y_{n-1}$. The quantities $\epsilon_{1}, \epsilon_{2}$ and $\epsilon_{3}$ are the moduli of 
continuity of $u_{x x}, u_{x y}$ and $u_{y y}$, respectively. In the same way we obtain for $y>0$, the estimate

$$
\left|L u-L_{h} u\right| \leqq K \epsilon_{1}+\left(1+\frac{h}{4}|b|\right) \epsilon_{3}+\frac{h}{2}|a|\left|\tilde{u}_{x x}\right|
$$

and for $y=0$,

$$
\left|L u-L_{h} u\right| \leqq \epsilon_{3}+\frac{\mathrm{h}}{2}|a|\left|\bar{u}_{x x}\right|+\frac{1}{2}|b|\left(h+y_{2}\right)\left|\bar{u}_{y v}\right| .
$$

By hypothesis, $K, a, b, u_{x x}$ and $u_{y y}$ are uniformly bounded on $\bar{D}$. Moreover, as $h \rightarrow 0, y_{2}, \epsilon_{1}$ and $\epsilon_{3}$ tend to zero, uniformly on $\bar{D}$. Hence (2.9) and (2.10) imply that $L_{h} u$ tends uniformly to $L u$ as $h \rightarrow 0$, for $y \geqq 0$. For $y<0$ we must evaluate the coefficients appearing in the estimate (2.8). For this it will be convenient to introduce the inverse function to $G$. More precisely, if $x=G(y)$ is the equation of the characteristic of the family (1.2b) passing through the origin, then we can express it also as

$$
y=-H(x) \text {. }
$$

The relation between $H$ and $K$ can be obtained by differentiating (2.11) to obtain

$$
H^{\prime}(x)=[-K(y)]^{-1 / 2}
$$

at each point $(x, y)$ on the curve (2.11). Hence $H$, which is defined for $0 \leqq x \leqq\left(x_{B}-x_{A}\right) / 2$, is an increasing function with $H(0)=0$, and $H^{\prime}$ is a decreasing function with $H^{\prime}(0)=+\infty$. That is, $H(x)>0, H^{\prime}(x)>0$ and $H^{\prime \prime}(x)$ $<0$ for $x>0$. Moreover, since we assume that $K$ has three continuous derivatives, $H$ will have four continuous derivatives for $x>0$.

Let us now return to (2.8). First we note that

$$
\lambda_{n}=y_{n}-y_{n-1}=H\left(\frac{n h}{2}\right)-H\left(\frac{n h-h}{2}\right)=\frac{h}{2} H^{\prime}\left(\frac{n h-\theta h}{2}\right),
$$

with $0<\theta<1$. Since $H(x) \rightarrow 0$ as $x \rightarrow 0$ we may choose $\delta>0$ so small that $H(\delta)<\epsilon / 2$. Then for all $n$ and $h$ such that $n h / 2 \leqq \delta$, we have $\lambda_{n}<\epsilon$. On the other hand, for $x \geqq \delta / 2, H^{\prime}(x)$ is uniformly bounded. Therefore, we may choose $h$ so small that for $n h / 2>\delta, \lambda_{n}<\epsilon$. Hence for all $n, \lambda_{n} \rightarrow 0$ uniformly as $h \rightarrow 0$. Using the relations (2.12) and (2.13) we find that

$$
K\left(-y_{n}\right)+\frac{h^{2}}{4 \lambda_{n} \lambda_{n+1}}=K\left(-y_{n}\right)+\left[-K\left(-y_{n-\alpha}\right)\right]^{1 / 2}\left[-K\left(-y_{n+\beta}\right)\right]^{1 / 2}
$$

where $y_{n-1}<y_{n-\alpha}<y_{n}<y_{n+\beta}<y_{n+1}$. Therefore since $K$ is continuous, the expression $h^{2} / 2 \lambda_{n} \lambda_{n+1}$ is uniformly bounded as $h \rightarrow 0$ and 


$$
K\left(-y_{n}\right)+\frac{h^{2}}{4 \lambda_{n} \lambda_{n+1}} \rightarrow 0
$$

as $h \rightarrow 0$. Also, since $\lambda_{n}>\lambda_{n+1}$, we have

$$
\frac{\lambda_{n}^{2}+\lambda_{n+1}^{2}}{2\left(\lambda_{n}+\lambda_{n+1}\right)}<\frac{\lambda_{n}}{2} .
$$

Hence, each term on the right side of (2.8) can be made arbitrarily small by choosing $h$ sufficiently small. This completes the proof of the theorem.

3. Maximum principles. To establish a maximum principle for the difference equation on the region $D_{h}$, we first prove maximum principles on each of the regions $D_{h}^{-}$and $D_{h}^{+}$separately. Let us take as the boundary of $D_{h}^{-}$the set $\Gamma_{h}^{-} \cup \gamma_{h}$, and as the boundary of $D_{h}^{+}$the set $\Gamma_{h}^{+} \cup \gamma_{h}$. We then denote by $\bar{D}_{h}^{-}$the set $D_{h}^{-}$plus its boundary, and by $\bar{D}_{h}^{+}$the set $D_{h}^{+}$plus its boundary.

From (2.2) we note that each point $\left(x,-y_{n}\right)$ of $\bar{D}_{h}^{-}$may be uniquely represented by a pair of indices $(k, n)$, where the first denotes the negatively sloping characteristic and the second the ordinate corresponding to the point. In this notation the difference operator (2.3) takes the form

$$
\begin{aligned}
L_{h} U_{k, n}=\frac{1}{\lambda_{n} \lambda_{n+1}}\left[\left(1-A_{k, n}\right) U_{k, n-1}+\left(1+A_{k, n}\right) U_{k-1, n+1}\right. \\
\left.-\left(1+\alpha_{k, n}\right) U_{k-1, n}-\left(1-\alpha_{k, n}-\gamma_{k, n}\right) U_{k, n}\right],
\end{aligned}
$$

in which we have defined

$$
\begin{aligned}
A_{k, n} & =\frac{\lambda_{n}-\lambda_{n+1}}{\lambda_{n}+\lambda_{n+1}}-\frac{\lambda_{n} \lambda_{n+1}}{\lambda_{n}+\lambda_{n+1}} b\left(x,-y_{n}\right), \\
\alpha_{k, n} & =\frac{\lambda_{n} \lambda_{n+1}}{h} a\left(x,-y_{n}\right), \\
\gamma_{k, n} & =\lambda_{n} \lambda_{n+1} c\left(x,-y_{n}\right) .
\end{aligned}
$$

TheOREM 3.1. Let $L_{h} U \geqq 0$ on $D_{h}^{-}$and

$$
U_{0, n+1} \leqq U_{0, n} ; \quad n \geqq 1 .
$$

Assume that the conditions

(3.9) $A_{k, n}-\alpha_{k, n}>0$,

(3.10) $A_{k+1, n-1}-A_{k, n}-A_{k, n} A_{k+1, n-1}-\alpha_{k+1, n-1}+\alpha_{k, n}+\alpha_{k, n} \alpha_{k+1, n-1}$

$$
+\left(1+\alpha_{k+1, n-1}\right) \gamma_{k, n} \geqq 0,
$$


are satisfied at all points $(k, n)$ of $D_{h}^{-}$. Then the maximum of $U$ on $\bar{D}_{h}^{-}$, if nonnegative, is attained on the boundary. If $\gamma_{k, n} \equiv 0$, then the result holds without the requirement that the maximum be non-negative.

Proof. Assume that the maximum of $U$ occurs at an interior point $(k, n)$, and that $U_{k, n}$ is greater than the maximum of $U$ on the boundary. We consider the set of points in $\bar{D}_{h}^{-}$which lie on the positively sloping characteristics passing through the points $(k, n)$ and $(k, n-1)$. Using (3.1) to write $L_{h} U_{k, n}$ $\geqq 0$ we have

$$
\begin{aligned}
\left(1+\alpha_{k, n}\right)\left(U_{k-1, n+1}-U_{k-1, n}\right) & \geqq\left(1-A_{k, n}\right)\left(U_{k, n}-U_{k, n-1}\right) \\
& +\left(A_{k, n}-\alpha_{k, n}\right)\left(U_{k, n}-U_{k-1, n+1}\right)-\gamma_{k, n} U_{k, n} \geqq 0,
\end{aligned}
$$

the second inequality following from the conditions (3.6), (3.8) and (3.9), together with the assumption that $U_{k, n}$ is the maximum and is non-negative. Next we assert that

$$
\begin{aligned}
\left(1+\alpha_{k-j+1, n+j-1}\right)\left(U_{k-j, n+j}\right. & \left.-U_{k-j, n+j-1}\right) \\
& \geqq(A-\alpha)_{k-j+1, n+j-1}\left(U_{k, n}-U_{k-j, n+j}\right) \geqq 0,
\end{aligned}
$$

for $j=1,2, \cdots, k$. The second inequality of (3.11) follows from (3.9) and the assumption that $U_{k, n}$ is the maximum. The first inequality we have proved for $j=1$, hence we proceed by induction. We will show that if (3.11) holds for any $j$ with $1 \leqq j \leqq k-1$, then it holds also for $j+1$. From $L_{h} U_{k-j, n+j}$ $\geqq 0$, we have

$$
\begin{array}{r}
\left(1+\alpha_{k-j, n+j}\right)\left(U_{k-j-1, n+j+1}-U_{k-j-1, n+j}\right) \geqq\left(1-A_{k-j, n+j}\right)\left(U_{k-j, n+j}-U_{k-j, n+j-1}\right) \\
+(A-\alpha-\gamma)_{k-j, n+j} U_{k-j, n+j}-(A-\alpha)_{k-j, n+j} U_{k-j-1, n+j+1} .
\end{array}
$$

Multiplying (3.11) by the quantity

$$
(A-\alpha-\gamma)_{k-j, n+j} /(A-\alpha)_{k-j+1, n+j-1}
$$

and adding the resulting inequality to the above inequality, we obtain

$$
\begin{aligned}
& \left(1+\alpha_{k-j, n+j}\right)\left(U_{k-j-1, n+j+1}-U_{k-j-1, n+j}\right) \\
& \geqq(A-\alpha)_{k-j, n+j}\left(U_{k, n}-U_{k-j-1, n+j+1}\right)-\gamma_{k-j, n+j} U_{k, n} \\
& \quad+\left\{\left(1-A_{k-j, n+j}\right)-(A-\alpha-\gamma)_{k-j, n+j}\left(\frac{1+\alpha}{A-\alpha}\right)_{k-j+1, n+j-1}\right\} \\
& \cdot\left(U_{k-j, n+j}-U_{k-j, n+j-1}\right) .
\end{aligned}
$$

The second term on the right side is non-negative by (3.6) and the assumption that $U_{k, n}$, the maximum, is non-negative. In the last term on the right side, the first factor is non-negative by (3.9) and (3.10), while the second factor is non-negative by the induction hypothesis (3.11). Thus (3.11) holds for $j+1$ and hence for $j=1,2, \cdots, k$.

For $j=k$, the first inequality of (3.11) can be written 


$$
U_{0, n+k}-U_{0, n+k-1} \geqq\left(\frac{A-\alpha}{1+\alpha}\right)_{1, n+k-1}\left(U_{k, n}-U_{0, n+k}\right) .
$$

Since it was assumed that the maximum does not occur on the boundary, the second factor on the right side is positive. From this we conclude that $U_{0, n+k}>U_{0, n+k-1}$ for some $k \geqq 1, n \geqq 1$, which contradicts the hypothesis (3.5). Hence the maximum must be attained on the boundary. The result for $\gamma_{k, n} \equiv 0$ follows by setting $\gamma_{k, n} \equiv 0$ in the proof.

Let us now consider the region $D_{h}^{+}$, for which we have the following maximum principle.

Theorem 3.2. Let $L_{h} U \geqq 0$ on $D_{h}^{+}$. Assume that the conditions

$$
\begin{aligned}
K(y)-h a(x, y) & >0 \\
1 \pm \frac{h}{2} b(x, y) & >0 \\
c & \leqq 0
\end{aligned}
$$

are satisfied on $D_{h}^{+}$. Then the maximum of $U$ on $\bar{D}_{h}^{+}$, if non-negative, is attained on the boundary. If $c \equiv 0$, then the result holds without requiring that the maximum be non-negative.

Proof. Let $M \geqq 0$ denote the maximum value of $U$ on $\bar{D}_{h}^{+}$and suppose that $M$ is greater than the maximum of $U$ on the boundary. Then there exists a point $(x, y)$ in $D_{h}^{+}$at which $U=M$, and for at least one of its four neighboring points $U<M$. Then, referring to the equation (2.4), it follows from the conditions (3.12), (3.13) and (3.14) that $L_{h} U(x, y)<0$, contrary to hypothesis. Thus the maximum must be attained on the boundary.

Corollary. Let $L_{h} U \geqq 0$ on $D_{h}^{+}, U \leqq 0$ on $\Gamma_{h}^{+}$and $U \leqq M$ on $\gamma_{h}$ with $M>0$. Suppose the conditions (3.12), (3.13) and (3.14) are satisfied on $D_{h}^{+}$. If $(x, 0)$ is a point of $\gamma_{h}$ at which $U=M$, then $U(x, 0)>U(x, h)$.

Proof. By Theorem 3.2, $U \leqq M$ throughout $D_{h}^{+}$. If $(x, h)$ is a boundary point of $\bar{D}_{h}^{+}$, then $U(x, h) \leqq 0$ by hypothesis, and the theorem is trivially satisfied. Therefore we can assume that $(x, h)$ is in $D_{h}^{+}$. Suppose that $U(x, h)$ $=M$. Then if $c(x, h)<0$ we find immediately from (2.4) that $L_{h} U(x, h)<0$, contrary to hypothesis. On the other hand, if $c(x, h)=0$, we must have $L_{h} U(x, h)=0$ which implies that $U=M$ at each of the four neighbors of the point $(x, h)$. In particular, $U(x, 2 h)=M$. Repeating this argument a finite number of times, we either contradict the assumption that $L_{h} U \geqq 0$ on $D_{h}^{+}$, or we obtain $U=M$ at some point of $\Gamma_{h}^{+}$, contrary to hypothesis.

We may now state and prove the following maximum principle for the whole domain $D_{h}$.

Theorem 3.3. Let $L_{h} U \geqq 0$ on $D_{h}$ with $U$ satisfying (3.5) on $\Gamma_{h}^{-}$. Assume 
that the conditions (3.6) through (3.10) are satisfied on $D_{h}^{-}$, and the conditions (3.12) through (3.14) are satisfied on $D_{h}^{+}$. Furthermore, suppose that on $\gamma_{h}$,

$$
\begin{aligned}
a(x, 0) & \leqq 0, \\
1+\frac{h}{2} b(x, 0) & >0, \\
1-\frac{y_{2}}{2} b(x, 0) & >[0, \\
c(x, 0) & \leqq 0 .
\end{aligned}
$$

Then the maximum of $U$ on $\bar{D}_{h}$, if non-negative, is attained on the boundary. If $c \equiv 0$, then the result holds without the requirement that the maximum be nonnegative.

Proof. Let $M$ be the maximum value of $U$ on the boundary $\Gamma_{h}$. Then in order that the theorem be meaningful, we must require that $M \geqq 0$. Let $M_{1}$ be the maximum of $U$ on $\gamma_{h}$ and suppose that $M_{1}>M$. Then the function $V=U-M$ satisfies $L_{h} V \geqq 0$ on $D_{h}, V \leqq 0$ on $\Gamma_{h}$, and $V \leqq M_{1}-M$ on $\gamma_{h}$ with $M_{1}-M>0$. Let $(x, 0)$ be a point of $\gamma_{h}$ where $U=M_{1}$. Then by the corollary to Theorem 3.2, $V(x, h)<M_{1}-M$, which means that $U(x, h)<M_{1}$. Moreover, since $V$ also satisfies (3.5), we have $U \leqq M_{1}$ on $D_{h}^{-}$by Theorem 3.1. By virtue of the conditions (3.15) through (3.18), the formula (2.5) gives us $L_{h} U(x, 0)$ $<0$. This however contradicts the assumption that $L_{h} U \geqq 0$ on $D_{h}$. Hence we must have $M_{1} \leqq M$. Then by Theorems 3.1 and 3.2 we immediately obtain $U \leqq M$ throughout $D_{h}$.

Since our difference equation was obtained from the differential equation (1.1), it is desirable that the conditions (3.6) through (3.10) and (3.12) through (3.18) be translated into conditions on the coefficients of the differential equation. It is clear that because the coefficients are assumed to be continuous on $\bar{D}$, the conditions (3.14) and (3.18) are satisfied if and only if $c \leqq 0$ on $\bar{D}^{+}$, while (3.13), (3.16) and (3.17) are satisfied if $h$ is sufficiently small. If we also assume that there is a $\delta>0$, such that $a(x, y) \leqq 0$ for $0 \leqq y \leqq \delta$, then the conditions (3.12) and (3.15) will be satisfied for $h$ sufficiently small, since $K$ is uniformly positive for $y \geqq \delta$. The conditions in $D_{h}^{-}$are investigated in the following.

THEOREM 3.4. If

$$
c \leqq 0 \text { on } \bar{D}^{-}
$$

and

$$
y a(x, y)[-K(y)]^{-1 / 2} \rightarrow 0 \text { as } y \rightarrow 0,
$$

uniformly on $\bar{D}^{-}$, then for h sufficiently small, the conditions (3.6), (3.7) and (3.8) are satisfied. 
Proof. (3.6) follows immediately from (3.19) and the continuity of $c$. Recalling the definition of $\alpha_{k, n}$, we have

$$
\left|\alpha_{k, n}\right|=\left|\frac{\lambda_{n} \lambda_{n+1}}{h} a\left(x,-y_{n}\right)\right| \leqq\left|y_{n} a\left(x,-y_{n}\right)\left[-K\left(-y_{n}\right)\right]^{-1 / 2}\right|
$$

using (2.12) and (2.13). Therefore, by the condition (3.20), there exists a $\delta_{1}>0$ such that $1+\alpha_{k, n}>0$ for all $y_{n}$ with $y_{n} \leqq \delta_{1}$. For $y_{n}>\delta_{1}$, the quantity

$$
\lambda_{n+1} / h \leqq \frac{1}{2}\left[-K\left(-y_{n}\right)\right]^{-1 / 2}
$$

is uniformly bounded. Thus since $\lambda_{n} \rightarrow 0$ as $h \rightarrow 0$ and $a$ is uniformly bounded on $\bar{D}^{-}, \alpha_{k, n} \rightarrow 0$ as $h \rightarrow 0$ for each $n$ such that $y_{n}>\delta_{1}$. This proves that (3.7) is satisfied on all of $D_{h}^{-}$provided only that $h$ is small.

Referring to (3.2) we may write

$$
1-A_{k, n}=\frac{2 \lambda_{n+1}}{\lambda_{n}+\lambda_{n+1}}\left[1+\frac{1}{2} \lambda_{n} b\left(x,-y_{n}\right)\right] \text {. }
$$

Since $\lambda_{n} \rightarrow 0$ as $h \rightarrow 0$ and $b$ is uniformly bounded on $\bar{D}^{-}$, (3.8) will be satisfied for $h$ sufficiently small.

Theorem 3.5. Let (3.20) hold on $D^{-}$, and let

$$
\frac{d}{d y}\left[(-K)^{1 / 2}\right]+a+b(-K)^{1 / 2}<0
$$

for $y<0$ on $\bar{D}^{-}$. Furthermore, assume that the function $H$ defined by (2.11) has a derivative of the form

$$
H^{\prime}(x)=x^{-\alpha} \tilde{H}(x)
$$

near $x=0$, where $0<\alpha<1$ and $\tilde{H}$ is a function having three continuous derivatives with $\tilde{H}(x) \geqq m>0$ for $x \geqq 0$. Then (3.9) is satisfied on $D_{h}^{-}$for $h$ sufficiently small.

Proof. From (3.20) we obtain for $n h$ sufficiently small

$$
y_{n} \geqq \frac{m}{1-\alpha}\left(\frac{n h}{2}\right)^{1-\alpha}, \quad n \geqq 1 ; \quad \lambda_{n}<\frac{M h}{2}\left(\frac{n h-h}{2}\right)^{-\alpha}, \quad n \geqq 2 ;
$$

where $\tilde{H}(x) \leqq M$. Furthermore, by writing

$$
\lambda_{n}-\lambda_{n+1}=\int_{(n-1) h / 2}^{n h / 2}\left[H^{\prime}(x)-H^{\prime}\left(x+\frac{h}{2}\right)\right] d x,
$$

and noting that $H^{\prime \prime}(x) \leqq(m \alpha / 2) x^{-\alpha-1}$ for $x$ sufficiently small, we find that for $n \geqq 2$, 


$$
\lambda_{n}-\lambda_{n+1}>\frac{m \alpha h^{2}}{8}\left(\frac{n h+h}{2}\right)^{-\alpha-1}
$$

Combining these estimates, we obtain for $n \geqq 2$,

$$
y_{n}\left(\lambda_{n}-\lambda_{n+1}\right) / \lambda_{n} \lambda_{n+1}>C_{0}>0,
$$

with $C_{0}$ a constant. On the other hand, we have

$$
\lambda_{1} \leqq \frac{M}{1-\alpha}\left(\frac{h}{2}\right)^{1-\alpha}, \quad \lambda_{2} \leqq \frac{M}{1-\alpha} h^{1-\alpha} .
$$

If $0 \leqq x \leqq h / 2,(3.20)$ yields the estimate

$$
H^{\prime}(x)-H^{\prime}\left(x+\frac{h}{2}\right) \geqq C_{1} x^{-\alpha},
$$

where $C_{1}$ is a positive constant. This in turn, implies that

$$
\lambda_{1}-\lambda_{2} \geqq \frac{C_{1}}{1-\alpha}\left(\frac{h}{2}\right)^{1-\alpha} .
$$

Combining these estimates we obtain

$$
y_{1}\left(\lambda_{1}-\lambda_{2}\right) / \lambda_{1} \lambda_{2}>C_{2}>0 \text {. }
$$

Therefore, for all $n \geqq 1$, the quantity $y_{n}\left(\lambda_{n}-\lambda_{n+1}\right) / \lambda_{n} \lambda_{n+1}$ is greater than a fixed positive number provided that $h$ and $y_{n}$ are sufficiently small. We also have

$$
\left|y_{n} \frac{\lambda_{n}+\lambda_{n+1}}{h} a\left(x,-y_{n}\right)\right| \leqq\left|\frac{y_{n} a\left(x,-y_{n}\right)}{2\left(-K\left(-y_{n}\right)\right)^{1 / 2}}\right| \frac{M}{m} \frac{2^{\alpha}}{1-\alpha} .
$$

Thus we may choose $h$ and $y_{n}$ so small, say $y_{n} \leqq \delta, \delta>0$, that (3.9) is satisfied.

For $y_{n}>\delta$, we choose $h$ so small that $y_{n-2} \geqq \delta / 2$. Then for $x \geqq(n h-2 h) / 2$ the function $H$ has three continuous and uniformly bounded derivatives. We may then apply Taylor's theorem to the expression $A_{k, n}-\alpha_{k, n}$ to obtain

$$
\begin{aligned}
A_{k, n}-\alpha_{k, n}= & -\frac{1}{\lambda_{n}+\lambda_{n+1}} \frac{h^{2}}{4}\left(H_{n}^{\prime}\right)^{3} \\
& \cdot\left\{\frac{H_{n}^{\prime \prime}}{\left(H_{n}^{\prime}\right)^{3}}+a\left(x,-y_{n}\right)+\frac{1}{H_{n}^{\prime}} b\left(x,-y_{n}\right)+O(h)\right\} \\
= & -\frac{1}{\lambda_{n}+\lambda_{n+1}} \frac{h^{2}}{4}\left\{-K\left(-y_{n}\right)\right\}^{-3 / 2}\left\{\frac{d}{d y}\left(-K\left(-y_{n}\right)\right)^{1 / 2}\right. \\
& \left.\quad+a\left(x .-y_{n}\right)+b\left(x,-y_{n}\right)\left(-K\left(-y_{n}\right)\right)^{1 / 2}+O(h)\right\},
\end{aligned}
$$


where $H_{n}=H(n h / 2)$. Because of (3.22), the expression in the brackets can be made negative by choosing $h$ small if $y_{n}>\delta$. This proves that (3.9) is satisfied for $y_{n}>\delta$.

It is of interest to ask how restrictive the condition (3.22) is on the function $H$. A partial answer is given by considering the class of functions

$$
K(y)=-(-y)^{m} ; \quad y \leqq 0,
$$

with $m$ a positive integer. The corresponding function $H^{\prime}$ is given by

$$
H^{\prime}(x)=\left(\frac{m+2}{2} x\right)^{-m /(m+2)}
$$

and thus this function satisfies (3.22) with $\alpha=m /(m+2)$ and $\tilde{H}(x)=(1-\alpha)^{\alpha}$.

THEOREM 3.6. If for $y<0$ on $\bar{D}^{-}$the condition

$$
\begin{aligned}
2(-K)^{1 / 4} \frac{d^{2}}{d y^{2}} & \left\{(-K)^{-1 / 4}\right\}-2 \frac{d}{d y}\left\{(-K)^{-1 / 2}\right\} a-\frac{1}{2 K} a^{2} \\
& -a_{x}-(-K)^{-1 / 2} a_{y}-\frac{1}{2} b^{2}-(-K)^{1 / 2} b_{x}-b_{y}+2 c>0
\end{aligned}
$$

is satisfied, then for each $\delta>0$ and for $h$ sufficiently small, the condition (3.10) holds at each point of $D_{h}^{-}$where $y_{n} \geqq \delta$.

Proof. Fix $\delta>0$ and choose $h$ so small that if $y_{n} \geqq \delta$, then $y_{n-2} \geqq \delta / 2$. Application of Taylor's theorem then yields for the left side of (3.10),

$$
\begin{array}{r}
{\left[\left(\lambda_{n-1}+\lambda_{n}\right)\left(\lambda_{n}+\lambda_{n+1}\right)\right]^{-1} \frac{h^{4}}{8}\left(H_{n}^{\prime}\right)^{4}\left[-2\left(H_{n}^{\prime}\right)^{-3 / 2} \frac{d^{2}}{d x^{2}}\left(H_{n}^{\prime}\right)^{-1 / 2}+2\left(H_{n}^{\prime}\right)^{-1} H_{n}^{\prime \prime} a\right.} \\
\left.+\frac{1}{2}\left(H_{n}^{\prime}\right)^{2} a^{2}-a_{x}-H_{n}^{\prime} a_{y}-\frac{1}{2} b^{2}-\left(H_{n}^{\prime}\right)^{-1} b_{x}-b_{y}+2 c+O(h)\right]
\end{array}
$$

where $a, a_{x}, a_{y}, b, b_{x}, b_{y}$ and $c$ denote values of these functions at $\left(x,-y_{n}\right)$. Using (2.12) to replace $H$ by $K$ in the above expression we obtain for the left side of (3.10),

$$
\begin{array}{r}
{\left[\left(\lambda_{n-1}+\lambda_{n}\right)\left(\lambda_{n}+\lambda_{n+1}\right)\right]^{-1} \frac{h^{4}}{8 K^{2}}\left\{2(-K)^{1 / 4} \frac{d^{2}}{d y^{2}}\left[(-K)^{-1 / 4}\right]-2 \frac{d}{d y}\left[(-K)^{-1 / 2}\right] a\right.} \\
\left.-\frac{1}{2 K} a^{2}-a_{x}-(-K)^{-1 / 2} a_{y}-\frac{1}{2} b^{2}-(-K)^{1 / 2} b_{x}-b_{y}+2 c+O(h)\right\} .
\end{array}
$$

Thus if we choose $h$ sufficiently small, the condition (3.23) will assure the validity of (3.10) for $y_{n} \geqq \delta$.

It is of interest to observe that the conditions (3.19), (3.21) and (3.23) are essentially the conditions under which the solution of the differential 
equation (1.1) in $D^{-}$satisfies a maximum principle, as found by Agmon, Nirenberg and Protter [4].

4. The existence of the solution to the difference equation. For convenience, let us denote by the Conditions A the set of conditions under which the maximum principle, Theorem 3.3 , holds. That is, we denote by Conditions A:

(a) (3.6), (3.7), (3.8), (3.9) and (3.10) hold on $D_{h}^{-}$,

(b) (3.12), (3.13) and (3.14) hold on $D_{h}^{+}$,

(c) (3.15), (3.16), (3.17) and (3.18) hold on $\gamma_{h}$.

Theorem 4.1. Let the Conditions A be satisfied. Then there exists a unique solution $U$ to the difference equation (2.6) on $D_{h}$ which satisfies the boundary conditions (2.7), for any given values of $f, \phi_{1}$ and $\phi_{2}$.

Proof. Let $V$ be a solution of the homogeneous system of equations,

$$
\begin{aligned}
L_{h} V & =0 \text { on } D_{h}, \\
V & =0 \text { on } \Gamma_{h} .
\end{aligned}
$$

Then by Theorem $3.3, V \leqq 0$ on $D_{h}$. But we may also apply Theorem 3.3 to the function $-V$ to obtain $V \geqq 0$ on $D_{h}$. Thus $V$ must vanish identically on the whole domain $D_{h}$; i.e., the homogeneous system has only the trivial solution $V \equiv 0$. But this implies that the system (2.6) and (2.7) has a unique solution for arbitrary right sides, i.e., for arbitrary values of $f, \phi_{1}$ and $\phi_{2}$.

We now show that the system of linear equations (2.6) and (2.7) can be solved by means of the Gauss-Seidel iteration procedure. For this we number the $P$ points of $D_{h}$ in the following order. First we take the points of $D_{h}^{+}$with the largest ordinate and number them in any order. Then we number the points on the next row down in any order, and continue this process until we have numbered all the points of $D_{h}^{+}$and $\gamma_{h}$. Next we number the points $(1, n)$ of $D_{n}^{-}$in order of increasing $n$, then the points $(2, n)$ in order of increasing $n$, and so on. If we denote the value of $U$ at the point $i(i=1,2, \cdots, P)$ by $U_{i}$, and solve each of the equations

$$
L_{h} U_{i}=f_{i}
$$

for $U_{i}$, we obtain a linear system of equations of the form

$$
U_{i}=\sum_{j=1}^{i-1} r_{i j} U_{j}+\sum_{j=i+1}^{P} r_{i j} U_{j}+s_{i} ; \quad i=1,2, \cdots, P .
$$

In the Gauss-Seidel procedure, an arbitrary zeroth order approximation $U_{i}^{(0)}, i=1,2, \cdots, P$, to the solution is chosen, and successive approximations are calculated by the formula

$$
U_{i}^{(m+1)}=\sum_{j=1}^{i-1} r_{i j} U_{j}^{(m+1)}+\sum_{j=i+1}^{P} r_{i j} U_{j}^{(m)}+s_{i}
$$


where $U_{i}^{(m)}$ denotes the $m$ th approximation to $U_{i}$.

Theorem 4.2. Let the Conditions A be satisfied. Then for $h$ sufficiently small and for arbitrary values $U_{i}^{(0)}, i=1,2, \cdots, P, U_{i}^{(m)} \rightarrow U_{i}$ as $m \rightarrow \infty$.

Proof.If we let $V_{i}^{(m)}$ be the error in the $m$ th approximation,

$$
V_{i}^{(m)}=U_{i}-U_{i}^{(m)},
$$

then the error terms satisfy the homogeneous system

$$
V_{i}^{(m+1)}=\sum_{j=1}^{i-1} r_{i j} V_{j}^{(m+1)}+\sum_{j=i+1}^{P} r_{i j} V_{j}^{(m)} .
$$

That is, $V_{i}^{(m)}$ can be considered the $m$ th approximation to the solution of the homogeneous system (4.1), starting from an arbitrary zeroth order approximation. Assume that

$$
V_{i}^{(m)} \leqq M ; \quad i=1,2, \cdots, P,
$$

for some $M>0$. Then referring to (2.4), we see that since the point $(x, y)$ which corresponds to $i=1$ is on the highest row of $D_{h}^{+}$, the value $V_{1}^{(m+1)}$ is related to the values of $V^{(m)}$ at $(x-h, y),(x+h, y)$ and $(x, y-h)$ at most. Furthermore, the conditions (3.12) and (3.14) imply that the coefficient of $U(x, y)$ in $(2.4)$ is negative. Hence we have

$$
V_{1}^{(m+1)} \leqq\left\{1-\frac{1+h b / 2-h^{2} c}{2 K+2-h a-h^{2} c}\right\} M .
$$

By choosing $h$ sufficiently small, the fraction on the right side can be made greater than a fixed number $\rho$ with $0<\rho<1$. We conclude that

$$
V_{1}^{(m+1)} \leqq(1-\rho) M
$$

for $h$ sufficiently small. By induction, this implies that for any point on the highest row of $D_{h}$ we have the estimate

$$
V_{i}^{(m+1)} \leqq(1-\rho) M .
$$

We now consider the first point in the next row down, say the $k$ th point. $V_{k}^{(m+1)}$ is related to the value of $V^{(m+1)}$ at the point immediately above (if that point belongs to $D_{h}$ ) and to the values of $V^{(m)}$ at the other adjacent points. Therefore, (2.4) gives us

$$
V_{k}^{(m+1)} \leqq\left(1-\rho^{2}\right) M .
$$

By induction we may conclude that this relation holds on the entire second row. Continuing this process, we arrive at the estimate 


$$
V_{i}^{(m+1)} \leqq\left(1-\rho^{r}\right) M,
$$

at each point of $D_{h}^{+}$, where $r$ is the number of rows in $D_{h}^{+}$. On $\gamma_{h}$, we use (2.5) to obtain

$$
V_{i}^{(m+1)} \leqq\left(1-\rho_{0}^{r+1}\right) M
$$

where $0<\rho_{0} \leqq \rho$. Hence, by virtue of (4.3), (4.4) also holds on $D_{h}^{+}$.

Due to the manner in which the points of $D_{h}^{-}$were ordered, the second sum does not appear in the formula (4.2) for $V_{i}^{(m+1)}$. But this means that $V_{i}^{(m+1)}$ satisfies the difference equation

$$
L_{h} V_{i}^{(m+1)}=0
$$

on $D_{h}^{-}$, with $V^{(m+1)} \equiv 0$ on $\Gamma_{h}^{-}$. Therefore, by Theorem 3.1 , the maximum of $V^{(m+1)}$ is attained on the boundary of $D_{h}^{-}$. That is, the bound (4.4) holds throughout $D_{h}^{-}$, and therefore throughout $D_{h}$. Since we may carry out the same procedure for $-V_{i}^{(m+1)}$, we obtain finally,

$$
\left|V_{i}^{(m+1)}\right| \leqq\left(1-\rho_{0}^{r+1}\right) M .
$$

Thus if we now choose an arbitrary zeroth order approximation $U_{i}^{(0)}$, then

$$
\left|V_{i}^{(m)}\right| \leqq\left(1-\rho_{0}^{r+1}\right)^{m} M_{0}
$$

where

$$
M_{0}=\max _{i}\left|U_{i}-U_{i}^{(0)}\right| .
$$

Hence this shows that $V_{i}^{(m)} \rightarrow 0$ as $m \rightarrow \infty$.

5. A priori bounds. The maximum principle may also be employed to establish a priori bounds for functions defined on $\bar{D}_{h}$.

THEOREM 5.1. Let the Conditions A be satisfied. Let $U$ be any function defined on $\bar{D}_{h}$ such that the quantities

$$
B_{1}=\max _{\Gamma_{h}}|U|, \quad B_{2}=\max _{\Gamma_{\bar{h}}^{-}}\left|U_{\bar{\tau}}\right|, \quad B_{\mathbf{z}}=\max _{D_{\mathbf{h}}}\left|L_{h} U\right|,
$$

are finite, where

$$
U_{\bar{\tau}_{0, n}}=\left(U_{0, n}-U_{0, n+1}\right) / \lambda_{n+1} .
$$

Then for $h$ sufficiently small,

$$
|U| \leqq B_{1}+C\left(B_{2}+B_{8}\right)
$$

on $D_{h}$, where $C$ is a finite, non-negative constant depending only on the domain $D$ and the functions $b$ and $c$. 
Proof. Let

$$
Y=\sup _{D}|y| \text {. }
$$

We now define a mesh function $E$ on $D_{h}$ by the equations

$$
\begin{aligned}
E\left(-y_{n}\right) & =\left(1-\mu \lambda_{n}\right) E\left(-y_{n-1}\right) \text { on } \overline{D_{h}^{-}} \\
E(y) & =(1+2 \mu h) E(y-h) \text { for } y=n h ; \quad n=1,2, \cdots \\
E(0) & =e^{2 \mu Y}
\end{aligned}
$$

where $\mu$ is a positive constant to be chosen. Let us choose $h$ so small that $\mu h \leqq 1 / 2$ and $\mu \lambda_{n} \leqq 1 / 2$ for all $n$. Then $E$ is a positive, nondecreasing function of $y$. Therefore, since on $D_{h}^{+}$we have

$$
E(n h)=(1+2 \mu h)^{n} e^{2 \mu Y} \leqq e^{4 \mu Y},
$$

$E$ is uniformly bounded on $D_{h}$, independent of $h$. To find a lower bound for $E$, we first note that due to our choice of $h$ small, the inequalities

$$
1-\mu \lambda_{n} \geqq 1 /\left(1+2 \mu \lambda_{n}\right)>e^{-2 \mu \lambda_{n}}
$$

are satisfied. Then on $D_{h}^{-}$,

$$
E\left(-y_{n}\right)=e^{2 \mu Y} \prod_{i=1}^{n}\left(1-\mu \lambda_{i}\right)>1,
$$

which implies that $E>1$ on $D_{h}$.

If in addition to requiring that $\mu h \leqq 1 / 2, \mu \lambda_{n} \leqq 1 / 2$, we also take $h$ so small that

$$
1 / y_{2} \geqq b(x, 0) / 2 \text {, }
$$

we may write as a lower bound for $L_{h} E$ on $D_{h}$,

$$
L_{h} E \geqq \mu^{2}-3|b| \mu+2 c .
$$

Finally, on $\Gamma_{h}^{-}$,

$$
E_{\tau_{0, n}}=\mu E\left(-y_{n}\right) \geqq \mu .
$$

Therefore we may choose $\mu$ so large that for $h$ small we have $L_{h} E \geqq 1$ on $D_{h}$ and $E_{\bar{r}} \geqq 1$ on $\Gamma_{h}^{-}$.

We now define functions $V$ and $W$ on $D_{h}$ by

$$
\begin{aligned}
& V=U+B_{1}+\left(B_{2}+B_{3}\right) E, \\
& W=-U+B_{1}+\left(B_{2}+B_{3}\right) E .
\end{aligned}
$$

We then see easily that $L_{h} V \geqq 0$ and $L_{h} W \geqq 0$ on $D_{h}$, and $V_{\bar{\tau}} \geqq 0$ and $W_{\bar{\tau}} \geqq 0$ on $\Gamma_{h}^{-}$. Since $V \geqq 0$ and $W \geqq 0$ on $\Gamma_{h}$, the maxima of $V$ and $W$ are non-negative, 
and the maxima of both functions occur on the boundary, by Theorem 3.3. The bound (5.1) for $U$ then follows with $C=e^{4 \mu Y}-1$.

\section{Convergence theorems.}

TheOREM 6.1. Suppose that the differential equation (1.1) has a solution $u$ in $D$ which satisfies the boundary conditions (1.3), and assume that $u$ is of class $C^{2}(\bar{D})$. Assume that the Conditions A are satisfied for $h$ sufficiently small and let $U$ be the solution to the difference equation (2.6) with boundary conditions (2.7). Then $U \rightarrow u$ uniformly on $D_{h}$ as $h \rightarrow 0$.

Proof. Theorem 2.1 asserts that given $\epsilon>0$, there exists an $h_{0}>0$ such that on $D_{h}$

$$
\left|L u-L_{h} u\right|<\epsilon / 2 C
$$

for $h \leqq h_{0}, C$ being the fixed constant of Theorem 5.1. But since $L u=L_{h} U=f$ on $D_{h}$, this estimate may be written

$$
\left|L_{h}(U-u)\right|<\epsilon / 2 C \text {. }
$$

Furthermore, since $U=\phi_{1}$ on $\Gamma_{h}^{+}$and $u=\phi_{1}$ on $\Gamma^{+}$and $u$ and $\phi_{1}$ are continuous on $\bar{D}^{+}$, there exists an $h_{1}>0$ such that

$$
|U-u|<\epsilon / 2
$$

on $\Gamma_{h}^{+}$, for $h \leqq h_{1}$. Finally, on $\Gamma_{h}^{-}, U-u \equiv 0$. Hence, the bound (5.1) applied to $U-u$ yields at all points of $D_{h}$,

$$
|U-u|<\epsilon
$$

for $h \leqq h_{2}=\max \left(h_{0}, h_{1}\right)$.

For the case $K(y)=y$ and $a \equiv b \equiv c \equiv 0$, Filippov [2] has proved a convergence theorem with somewhat weakened conditions on the derivatives of $u$ at the boundary, provided that the curve $\Gamma^{+}$satisfies certain conditions. We will prove an analogous theorem for the equation (1.1), under similar conditions on $\Gamma^{+}$, and with additional restrictions on the function $K$.

Let us first extend the definition of the function $G$, defined for $y \leqq 0$ by equation (2.1), to positive values of $y$ by

$$
G(y)=\int_{0}^{y}[K(\eta)]^{1 / 2} d \eta ; \quad y>0 .
$$

We will say that the curve $\Gamma^{+}$satisfies Condition. $B$ if there exists an $x_{0}$ with $x_{A}<x_{0}<x_{B}$ such that for each real number $t$, the curve

$$
x-x_{0}=t G(y)
$$

intersects the curve $\Gamma^{+}$at only one point.

Lemma 6.1. Let $\Gamma^{+}$satisfy Condition B. Then if $0<\theta<1$, the transformation 


$$
\begin{aligned}
\bar{x} & =\theta\left(x-x_{0}\right)+x_{0}, \\
G(\bar{y}) & =\theta G(y) ;
\end{aligned}
$$$$
\operatorname{sgn} \bar{y}=\operatorname{sgn} y \text {, }
$$

maps each point $(x, y)$ of $\bar{D}$ into a point $(\bar{x}, \bar{y})$ of the interior $D$.

Proof. We note that $\bar{y}$ is uniquely defined by (6.3) since $G$ is a monotone function for $y>0$ and $y<0$.

Suppose $(x, y)$ lies in $\bar{D}$ with $y>0$. Then for some number $t$, the point $(x, y)$ lies on the curve (6.2). Therefore, since $\bar{y}<y$, the Condition B implies that $(\bar{x}, \bar{y})$ is a point of $D$.

For each point $(x, y)$ in $\bar{D}^{-}$we have,

$$
y \leqq 0, \quad x_{A}+G(y) \leqq x \leqq x_{B}-G(y) .
$$

Then the point $(\bar{x}, \bar{y})$ given by $(6.3)$ is also a point of $D$, since

$$
\bar{y} \leqq 0, \quad x_{A}+G(\bar{y})<\bar{x}<x_{B}-G(\bar{y}) .
$$

Let us define

$$
F_{\theta}(y)=[K(y) / K(\bar{y})]^{1 / 2} .
$$

Then for each fixed $\theta$ with $0<\theta<1, F_{\theta}$ is continuously differentiable and $F_{\theta}(y) \rightarrow 1$ as $\theta \rightarrow 1$ if $y \neq 0$. In the following theorem, we require that $F_{\theta}$ have smooth properties at $y=0$. We summarize the properties under the Conditions $\mathrm{C}$.

Conditions $C$ :

(a) For each fixed $\theta$ with $0<\theta<1, F_{\theta}$ is continuously differentiable on $\bar{D}$,

(b) $F_{\theta}(y) \rightarrow 1$ and $F_{\theta}^{\prime}(y) \rightarrow 0$ as $\theta \rightarrow 1$, uniformly on $\bar{D}$,

(c) For $y<0, y / \bar{y}$ is uniformly bounded.

We also need somewhat stronger Conditions $\mathrm{A}$ which we will denote by Conditions $A^{*}$ :

Conditions $\mathrm{A}$ hold and there is a $\delta>0$ such that

(a) (3.8) holds with $1-\delta$ on the right side,

(b) (3.9), (3.10) and (3.12) hold with $\delta$ on the right side.

THEOREM 6.2. Suppose that the differential equation (1.1) has a solution $u$ in $D$ which satisfies the boundary conditions (1.3), where $u$ is a function of class $C(\bar{D})$ and $C^{2}(D)$, with $u_{y}-(-K)^{1 / 2} u_{x}$ continuous on $D^{-} \cup \Gamma_{1}$. A ssume moreover that the Conditions: $\mathrm{A}^{*}$ are satisfied for all $h$ sufficiently small, and that the Conditions $\mathrm{B}$ and $\mathrm{C}$ and (3:20) are satisfied. Then the solution of the difference equation (2.6) with boundary conditions (2.7) tends uniformly to $u$ as $h \rightarrow 0$.

Proof. We define a new function $u_{\theta}$ by

$$
u_{\theta}(x, y)=u(\bar{x}, \bar{y}),
$$

where $(x, y)$ and $(\bar{x}, \bar{y})$ are related by (6.3). By Lemma 6.1 , Conditions $C$ and the assumption that $u$ is of class $C^{2}(D)$, we find that $u_{\theta}$ is of class $C^{2}(\bar{D})$ 
for each $\theta$ with $0<\theta<1$. Furthermore, since $(\bar{x}, \bar{y})$ tends uniformly to $(x, y)$ as $\theta \rightarrow 1$ and $u$ is of class $C(\bar{D})$, if $\epsilon>0$ is given, then there is a $\theta_{0}<1$ such that

$$
\left|u-u_{\theta}\right|<\epsilon / 3
$$

on $\bar{D}$ for each $\theta$ with $\theta_{0} \leqq \theta<1$.

Since $u$ is a solution of (1.1) on $D$, for each $\theta$ with $0<\theta<1$, the function $u_{\theta}$ satisfies the differential equation

(6.5) $\quad L_{\theta} u_{\theta}=K(y) u_{\theta_{x x}}+u_{\theta_{y y}}+a_{\theta}(x, y) u_{\theta_{x}}+b_{\theta}(x, y) u_{\theta_{y}}+c_{\theta}(x, y) u_{\theta}=f_{\theta}(x, y)$

on $\bar{D}$, where

$$
\begin{aligned}
& a_{\theta}(x, y)=\theta F_{\theta}^{2}(y) a(\bar{x}, \bar{y}), \\
& b_{\theta}(x, y)=\theta F_{\theta}(y) b(\bar{x}, \bar{y})-F_{\theta}^{\prime}(y) / F_{\theta}(y), \\
& c_{\theta}(x, y)=\theta^{2} F_{\theta}^{2}(y) c(\bar{x}, \bar{y}), \\
& f_{\theta}(x, y)=\theta^{2} F_{\theta}^{2}(y) f(\bar{x}, \bar{y}) .
\end{aligned}
$$

The Conditions $\mathrm{C}$ then imply that

$$
a_{\theta} \rightarrow a, \quad b_{\theta} \rightarrow b, \quad c_{\theta} \rightarrow c, \quad f_{\theta} \rightarrow f \quad \text { as } \theta \rightarrow 1,
$$

uniformly on $\bar{D}$. We next assert that if we define quantities $A_{\theta_{k, n}}, \alpha_{\theta_{k, n}}$ and $\gamma_{\theta_{k, n}}$ by replacing $a, b$ and $c$ in equations (3.2), (3.3) and (3.4) by $a_{\theta}, b_{\theta}$ and $c_{\theta}$, then for $h$ sufficiently small,

$$
A_{\theta_{k, n}} \rightarrow A_{k, n}, \quad \alpha_{\theta_{k, n}} \rightarrow \alpha_{k, n}, \quad \gamma_{\theta_{k, n}} \rightarrow \gamma_{k, n} \quad \text { as } \theta \rightarrow 1,
$$

uniformly on $D_{h}^{-}$. The first and third limits of (6.8) are immediate consequences of (6.7) since the quantities multiplying $b$ and $c$ in (3.2) and (3.4), respectively, are uniformly bounded for $h$ sufficiently small. For the second limit, we know from (2.13) that

$$
\lambda_{n} \lambda_{n+1} / h \leqq \frac{1}{2} \lambda_{n}\left[-K\left(-y_{n}\right)\right]^{-1 / 2} .
$$

Hence $\lambda_{n} \lambda_{n+1} / h$ is uniformly bounded for $y_{n} \geqq \delta>0$, and the second limit follows for $y_{n} \geqq \delta$. For $y_{n}<\delta$, we have

$$
\left|\alpha_{\theta k, n}\right|=\left|\frac{\lambda_{n} \lambda_{n+1}}{h} \theta F_{\theta}^{2}(y) a(\bar{x}, \bar{y})\right| \leqq\left|\frac{y_{n}}{2 \bar{y}} \theta F_{\theta}(y) \frac{\bar{y} a(\bar{x}, \bar{y})}{[-K(\bar{y})]^{1 / 2}}\right|,
$$

and the right side can be made arbitrarily small by making $y_{n}$ small, by virtue of the Conditions $C$ and (3.20). But from the proof of Theorem 3.4, we observe that the condition (3.20) also implies that $\alpha_{k, n} \rightarrow 0$ as $y_{n} \rightarrow 0$. Hence if we choose $\delta$ sufficiently small, the difference $\alpha_{k, n}-\alpha_{\theta_{k, n}}$ can be made arbitrarily small for $y_{n}<\delta$.

From (6.7) and (6.8) it follows that the Conditions A are satisfied by the 
coefficients of the difference approximation to $L_{\theta}$ provided that $h$ is small and $\theta$ is close to 1 , since we have assumed that the stronger Conditions $A^{*}$ are satisfied for all $h$ sufficiently small. Hence the difference problem corresponding to the differential equation (6.5) with boundary conditions

$$
u_{\theta}(x, y)=u(\bar{x}, \bar{y}) \text { for }(x, y) \text { on } \Gamma,
$$

has a unique solution $U_{\theta}$, if $h$ is small and $\theta$ is near 1 . Moreover, since $u_{\theta}$ is twice continuously differentiable on $\bar{D}$ for each $\theta$ with $0<\theta<1, U_{\theta}$ tends uniformly to $u_{\theta}$ as $h \rightarrow 0$, by Theorem 6.1. That is, there exist numbers $h_{1}>0$ and $\theta_{1}<1$ such that on $D_{h}$,

$$
\left|u_{\theta}-U_{\theta}\right|<\epsilon / 3
$$

for each $h$ and $\theta$ with $0<h \leqq h_{1}, \theta_{1} \leqq \theta<1$.

Let us now compare the solution $U$ of the difference approximation to $L u=f$ with the solution $U_{\theta}$ of the difference approximation to $L_{\theta} u_{\theta}=f_{\theta}$. We have

$$
L_{h}\left(U-U_{\theta}\right)=\left(f-f_{\theta}\right)=\left(a-a_{\theta}\right) U_{\theta_{x}}-\left(b-b_{\theta}\right) U_{\theta_{\nu}}-\left(c-c_{\theta}\right) U_{\theta},
$$

where $U_{\theta_{x}}$ and $U_{\theta_{y}}$ are difference quotients of $U_{\theta}$ defined in (2.3), (2.4) and (2.5). Since the functions $a_{\theta}$ and $b_{\theta}$ are independent of $h$, for each $h$ we may choose $\theta$ so close to 1 that $\left|a-a_{\theta}\right|<h^{2}$ and $\left|b-b_{\theta}\right|<h^{2}$. Hence, due to the uniform boundedness of $U_{\theta}$ on $\bar{D}_{h}$ for $h$ sufficiently small, it follows that for $h$ small

$$
L_{h}\left(U-U_{\theta}\right)<\epsilon / 12 C,
$$

where $C$ is the constant of Theorem 5.1. Furthermore, because $u$ is continuous on $\bar{D}$, it is clear that if $h$ is small and $\theta$ is near 1 ,

$$
\left|U-U_{\theta}\right|<\epsilon / 6
$$

on the boundary $\Gamma_{h}$. Likewise, by selecting $h$ and $\theta$ appropriately, it is possible to make

$$
\left|U_{\bar{\tau}}-U_{\theta_{\bar{\tau}}}\right|<\epsilon / 12 C,
$$

since $U=u$ and $U_{\theta}=u_{\theta}$ on $\Gamma_{1}$, and $d u_{\theta} / d y$, which is given by

$$
\frac{d u_{\theta}}{d y}(x, y)=\theta F_{\theta}(y)\left[u_{\bar{y}}(\bar{x}, \bar{y})-(-K(\bar{y}))^{1 / 2} u_{\bar{x}}(\bar{x}, \bar{y})\right]
$$

is continuous on $\Gamma_{1}$ and tends to $d u / d y$ as $\theta \rightarrow 1$. If we now apply Theorem 5.1 to the function $U-U_{\theta}$, we find that on $D_{h}$,

$$
\left|U-U_{\theta}\right|<\epsilon / 3
$$

for $h$ and $\theta$ such that $0<h \leqq h_{2}, \theta_{2} \leqq \theta<1$. Taken together, these estimates imply that given $\epsilon>0$, there is an $h_{0}>0$ such that if $0<h \leqq h_{0}$, then 


$$
|u-U|<\epsilon
$$

In one particular case, namely that in which the function $K$ is given by

$$
K(y)=|y|^{m} \operatorname{sgn} y
$$

for some positive integer $m$, the function $F_{\theta}$ takes on an especially simple form, with the result that the required conditions are easily verified. From (2.1) and (6.1) we obtain

$$
G(y)=\frac{2}{m+2}|y|_{(m+2) / 2} .
$$

Recalling that $G(\bar{y})=\theta G(y)$, we find that

$$
\bar{y}=\theta^{2 /(m+2)} y .
$$

Then we can easily calculate

$$
F_{\theta}(y)=\theta^{-m /(m+2)} .
$$

That is, $F_{\theta}$ depends only on $\theta$ and not on $y$. The Conditions $\mathrm{C}$ are clearly satisfied in this case.

In conclusion, it should be noted that the results are still valid for problems of the type:

(a) to solve $L u=f$ in $D^{+}$with $u$ given on $\Gamma^{+}$and $\bar{A} \bar{B}$,

(b) to solve $L u=f$ in $D^{-}$with $u$ given on $\Gamma_{1}$ and $\bar{A} \bar{B}$.

The maximum principles, a priori bounds and convergence theorems are applicable with obvious modifications in the hypotheses. Theorem 6.2 applies in either case (a) or (b) if the solution $u$ is assumed to be twice continuously differentiable on $D \cup \bar{A} \bar{B}$.

\section{BIBLIOGRAPHY}

1. F. Tricomi, Sulle equazioni linearn alle derivate parziali di $2^{\circ}$ ordine di tipo misto, Atti Accad. Naz. dei Lincei (5) vol. 14 (1923).

2. A. F. Filippov, On difference methods for the solution of the Tricomi problem, Izv. Akad. Nauk SSSR. Ser. Mat. vol. 21 (1957) pp. 73-88.

3. P. Germain and R. Bader, Sur quelques problèmes a l'équation de type mixte de Tricomi, Office Nationale d'Etudes et de Recherches Aéronautiques, Publication No. 54, 1952.

4. S. Agmon, L. Nirenberg and M. H. Protter, A maximum principle for a class of hyperbolic equations and applications to equations of mixed elliptic-hyperbolic type, Comm. Pure Appl. Math. vol. 6 (1953) pp. 445-470.

UNIVERSITY OF CALIFORNIA, Riverside, California 